
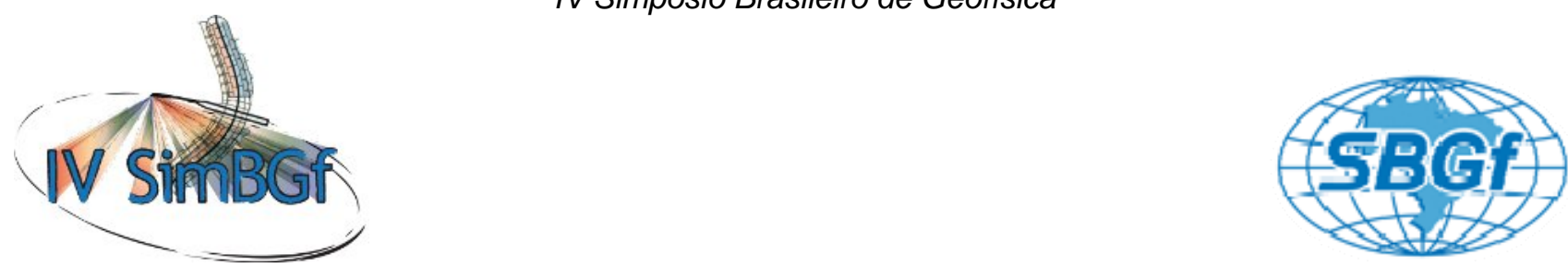

\title{
Simulação do perfil de Ressonância Magnética Nuclear utilizando perfis geofísicos de poços convencionais através da Lógica Fuzzy
}

Carlos Maximiano \& Abel Carrasquilla, UENF/CCT/LENEP - Macaé, RJ

Copyright 2008, SBGf - Sociedade Brasileira de Geofísica

Este texto foi preparado para a apresentação no IV Simpósio Brasileiro de Geofísica, Belém, 14 a 17 de novembro de 2010. Seu conteúdo foi revisado pelo Comitê Técnico do IV SimBGf, mas não necessariamente representa a opinião da SBGf ou de seus associados. É proibida a reprodução total ou parcial deste material para propósitos comerciais sem prévia autorização da SBGf.

\section{Resumo}

A técnica da Ressonância Magnética Nuclear (RMN) é atualmente utilizada na perfilagem geofísica de poços para fornecer informações petrofísicas dos fluídos livres, dos fluídos irredutíveis, da porosidade total, da porosidade efetiva e da permeabilidade. Isto permite uma melhor interpretação, o que conduz à conclusões práticas que facilitam a avaliação de hidrocarbonetos nos reservatórios. Neste trabalho, utilizamos a Lógica Fuzzy, na plataforma MATLAB, para sintetizar o perfil RMN, usando como entrada perfis convencionais de raios gama naturais, potencial espontâneo, caliper e resistividade. A sínteses do perfil RMN se justifica, pois ele é oneroso e não se realiza em todos os poços, mas, fornece essa série de características físicas importantes dos reservatórios que dificilmente é obtida por outros meios. Nossos resultados mostram que com a utilização do algoritmo ISODATA obtivemos um melhor ajuste nas curvas de porosidade (MPHI) e permeabilidade (MPERM), retirando o formato caixa que aparecia no perfil simulado e promovendo uma divisão de grupos mais criteriosa. Isto demonstrou a viabilidade do uso da Lógica Fuzzy na simulação de perfis RMN utilizando com entrada perfis de poço convencionais.

\section{Introdução}

A Ressonância Magnética Nuclear (RMN) é uma técnica que permite determinar propriedades de uma substância através do correlacionamento da energia absorvida contra a frequência, na faixa de megahertz $(\mathrm{MHz})$ do espectro eletromagnético, caracterizando-se como sendo uma espectroscopia. Usa as transições entre níveis de energia rotacionais dos núcleos componentes das espécies (átomos ou íons) contidas na amostra. Isso dá-se necessariamente sob a influência de um campo magnético e sob a concomitante irradiação de ondas de rádio na faixa de freqüências acima citada (Coates et al., 1999).

A técnica RMN é muito usada em medicina e em biologia faz já muito tempo, como meio de formar imagens internas de corpos humanos e de animais, bem como de seres microscópicos. $\mathrm{Na}$ industria do petróleo, o seu uso intenso começou nos anos 90 do século passado, como um meio de fornecer importantes informações petrofísicas dos reservatórios, sobre fluídos presentes, a porosidade e a permeabilidade dos reservatórios. Tratase de um perfil de poço bastante caro, que não se realiza em todos os poços, mas que, pelas características físicas valiosas dos reservatórios que oferece, se justifica a sua utilização (Asquith \& Gibson, 1982).

Pensando nessas propriedades do perfil de RMN, Richardson (1999) utilizou a técnica de inteligência artificial das redes neurais para sintetizar o perfil de RMN, utilizando como entrada perfis reais convencionais de poço como Caliper (CA), Raios Gama (GR), Potencial Espontâneo (SP) e Resistividade (Rt) de uma região localizada no Texas Oriental (Figura 1).

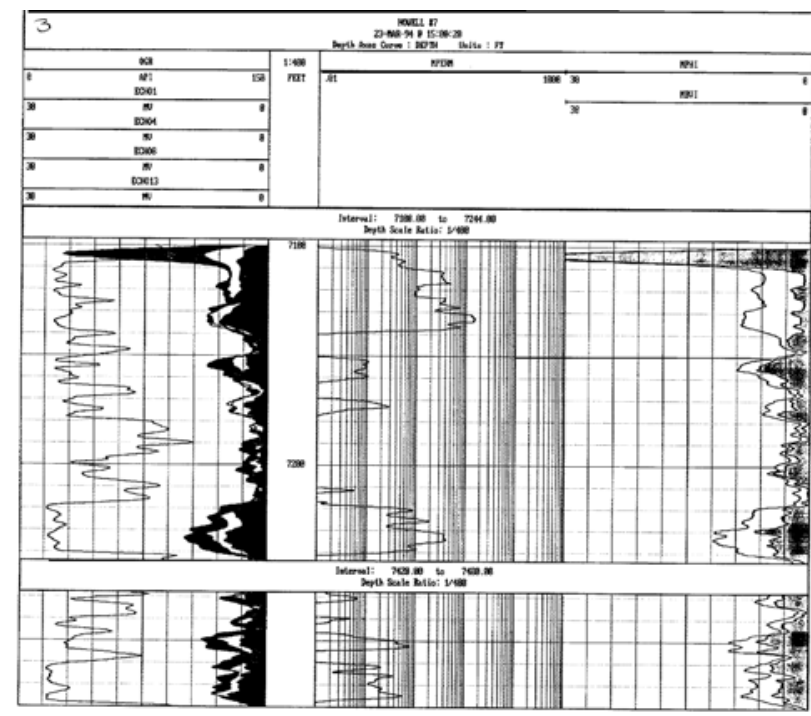

Figura 1. Perfis de poço reais e convencionais Caliper (CA), Raios Gama (GR), Potencial Espontâneo (SP) e Resistividade (Rt) de Texas Oriental.

Segundo Richardson (1999), a geologia da região que estudou é composta por arenitos deltaicos intercalados por folhelhos. Representados na forma de perfil, percebese essa intercalação nas curvas do perfil GR e um intenso desmoronamento na região em torno de $7.180 \mathrm{ft}$ representada pelo CA. Os perfis GR e SP apresentam regiões com baixos valores, indicando zonas de reservatórios nas profundidades de $7.135 \mathrm{ft}, 7.158 \mathrm{ft}, 7.175 \mathrm{ft}$, e nas regiões de 7.220 até $7.240 \mathrm{ft}$. O perfil de resistividade ILD indica altos valores para essas mesmas profundidades, o que também indica a presença dos reservatórios. As outras curvas de resistividade (RFOC e RILM) indicam valores mais baixos de resistividades dentro do reservatório, o que se deve a influência da invasão da lama de 
perfuração. As curvas ILD, RFOC e RILM, são os perfis de resistividades que serão utilizados como $\mathrm{Rt}$ neste trabalho.

No caso da porosidade, a simulação de Richardson (1999) com redes neurais deu os resultados mostrados na Figura 2, quanto que, para permeabilidade, os resultados são mostrados na Figura 3, considerando-se ambas como boas estimativas.

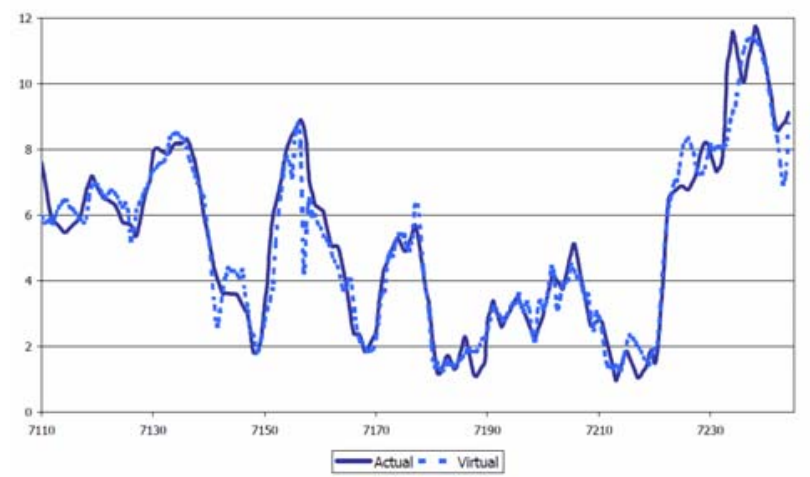

Figura 2. Resultados de Richardson (1999) para porosidade com dados de Texas Oriental.

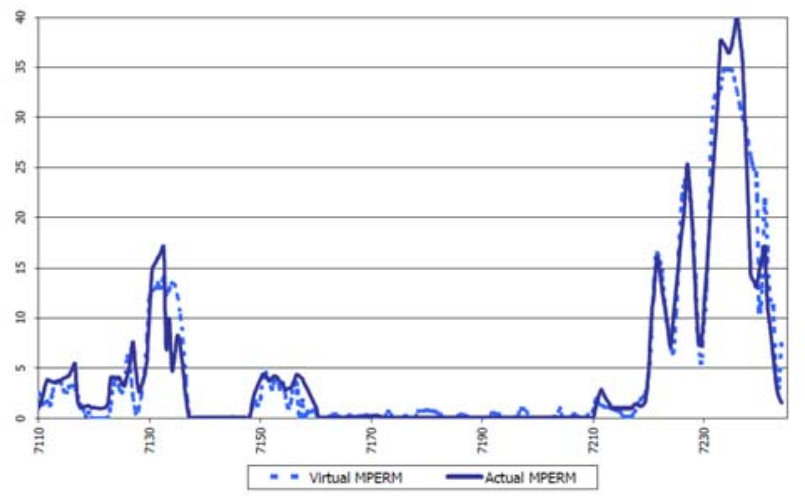

Figura 3. Resultados de Richardson (1999) para permeabilidade com dados de Texas Oriental.

No nosso trabalho, decidimos testar uma outra técnica de inteligência artificial, a Lógica Fuzzy, e comparar os resultados com os alcançados por Richardson (1999), quem utilizou redes neurais. A Lógica Fuzzy é uma extensão da lógica booleana que admite valores lógicos intermediários entre o falso (0) e o verdadeiro (1); por exemplo o valor médio talvez $(0,5)$. Isto significa que um valor lógico difuso é um valor qualquer no intervalo de valores entre 0 e 1. Este tipo de lógica engloba de certa forma conceitos estatísticos principalmente na área de Inferência (Zadeh et al., 1975). As implementações da Lógica Fuzzy permitem que estados indeterminados possam ser tratados por dispositivos de controle. Desse modo, é possível avaliar conceitos não - quantificáveis, como, por exemplo avaliar a temperatura (quente, morno, médio, etc.), 0 sentimento de felicidade(radiante, feliz, apático, triste, etc.) ou a veracidade de um argumento (correctíssimo, correcto, contraargumentativo, incoerente, falso, totalmente errôneo, etc.).

\section{Metodologia}

$\mathrm{Na}$ execução deste trabalho, inicialmente, digitalizamos os dados de perfis de poços convencionais (GR, SP, CA e Rt) do trabalho de Richardson (1999), já que não tínhamos eles no formato digital. Essa digitalização foi feita através da função impixel do software MATLAB (2008) para aquisição dos valores numéricos digitais que serviriam como entrada no algoritmo de simulação da lógica fuzzy, com a finalidade de gerar uma curva sintética RMN (Chapman, 2006). A seguir, o toolbox para regra fuzzy no MATLAB (2008) foi utilizado, para dar entrada das quatro variáveis (RG, CA, SP e RT), utilizadas neste estudo, que são os perfis de poço convencionais de Texas Oriental. Essas variáveis são informadas ao sistema de inferência definido por padrão como do tipo Mandami (1974). A seguir, processos como a definição da classe lingüística, os seus pontos de abrangência, as regras de inferência, a clusterização (agrupamento), a utilização do algoritmo isodata para auto-organizar os agrupamentos e a defuzificação, foram utilizados dentro da abordagem proposta (Figura 4). No final, se obtem como variáveis de saída de toda essa metodologia as curvas de permeabilidade (MPERM) e de porosidade (MPHI) sintéticas.

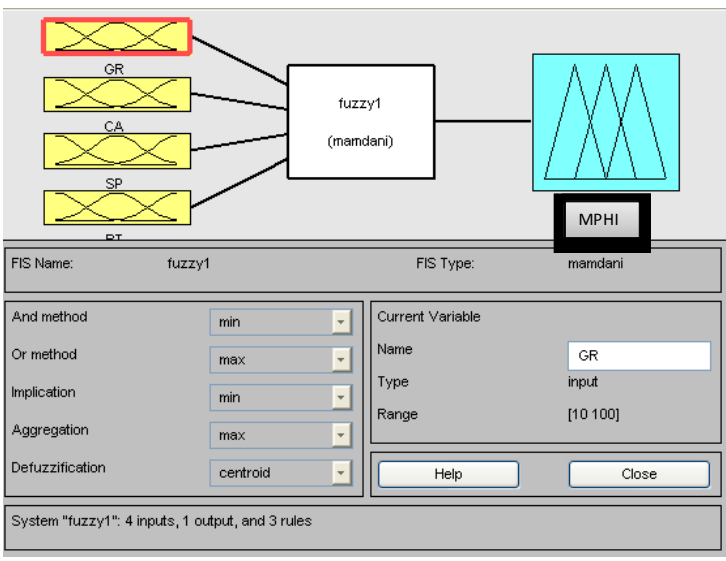

Figura 4. Toolbox da Lógica Fuzzy do MATLAB (2008).

No final, foi feita uma comparação dessa simulação com as curvas reais de permeabilidade (MPERM) e de porosidade (MPHI) do perfil de RMN de Texas Oriental do trabalho de Richardson (1999).

\section{Resultados}

A Figura 5 mostra as correlações cruzadas das variáveis de entradas dos perfis geofísicos convencionais (GR, SP, CA e Rt) da região do Texas Oriental. Observam-se grupos bem definidos (círculos pretos) quando são plotadas as variáveis de entradas GR $\times \mathrm{CA}$ e $\mathrm{GR} \times \mathrm{SP}$, entretanto, os gráficos CA x SP e SP $\times$ Rt mostram pontos mais espalhados. No estudo dos agrupamentos, 0 mesmo tipo de gráfico foi obtido para as correlações cruzadas desses perfis e as funções de permeabilidade e de porosidade, os quais não mostramos neste trabalho. 


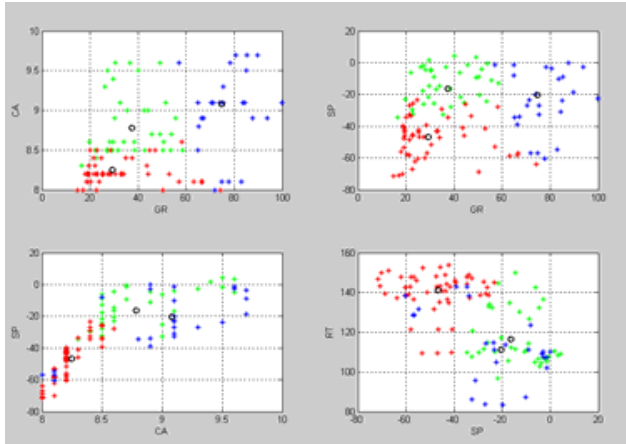

Figura 5. Correlações cruzadas dos perfis de poço de Texas Oriental.

Em seguida, forma realizadas três tentativas de sintetizar os perfis de permeabilidade e de porosidade, escolhendo as funções de pertinência de entrada para permeabilidade (Figura 6) e para porosidade (Figura 7), assim como as de saída para as funções da permeabilidade e da porosidade (Figura 8). Esta abordagem não deu bons resultados, pois as estimativas tanto para permeabilidade como para porosidades deram na forma de caixa e longe dos valores reais para esses parâmetros.
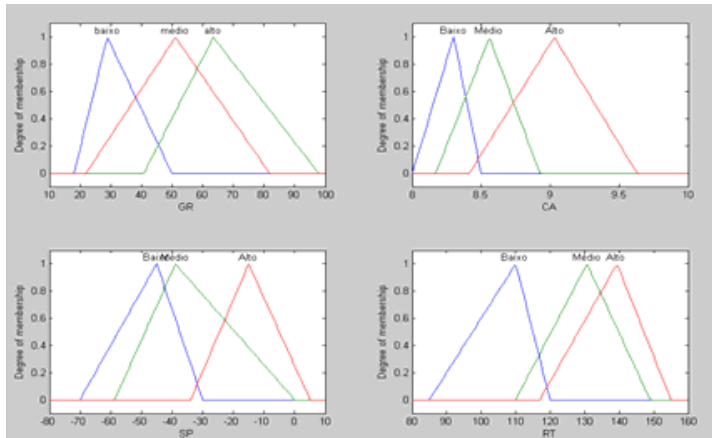

Figura 6. Funções de pertinência para as entradas da simulação de MPERM.

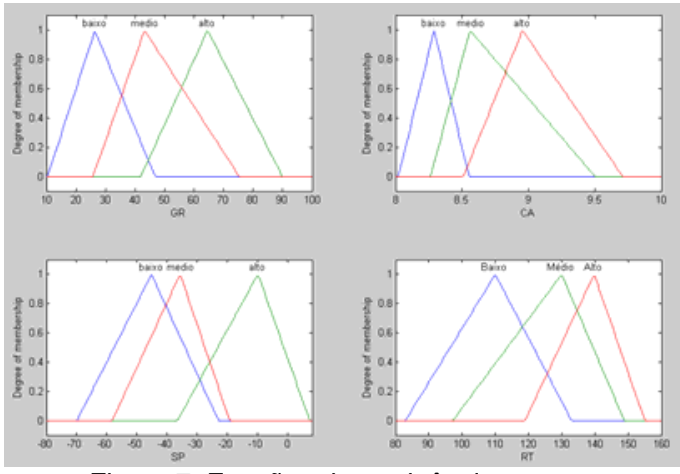

Figura 7. Funções de pertinência para as entradas da simulação de MPHI.

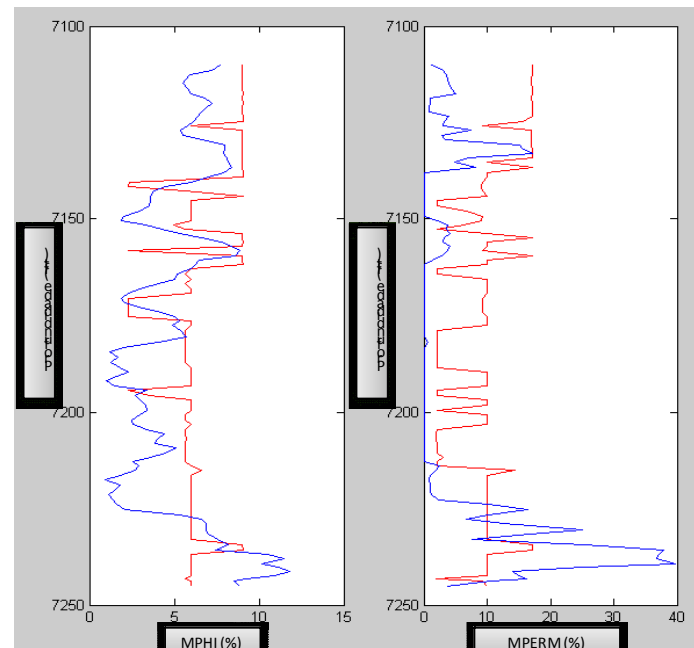

Figura 8. Resultados para as saídas fuzzy da simulação, em azul são os perfis de RMN reais (MPHI e MPERM), enquanto em vermelho são as curvas sintéticas dos mesmos perfis.

Esse problema foi resolvido com a eliminação da função de pertinência médio e utilização do algoritmo ISODATA do MATLAB (2008). Para exemplificar,na Figura 9 e na Figura 10 apresentamos os resultados dessa abordagem para o caso da permeabilidade.

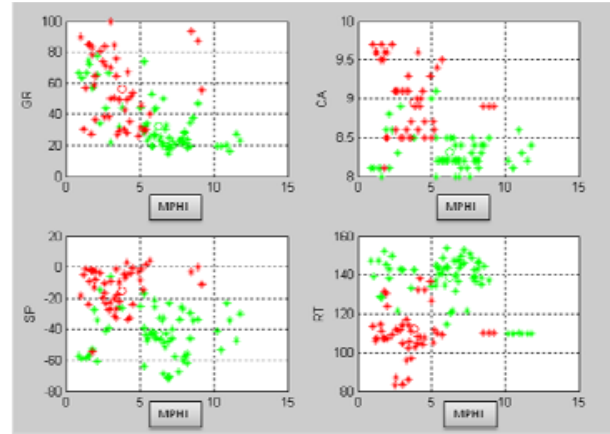

Figura 9. Variáveis de entrada (GR, CA, SP e Rt) versus variável de saída (MPHI), indicando a distribuição dos pontos pelos grupos, sendo os círculos vermelho e o verde os centros dos grupos.

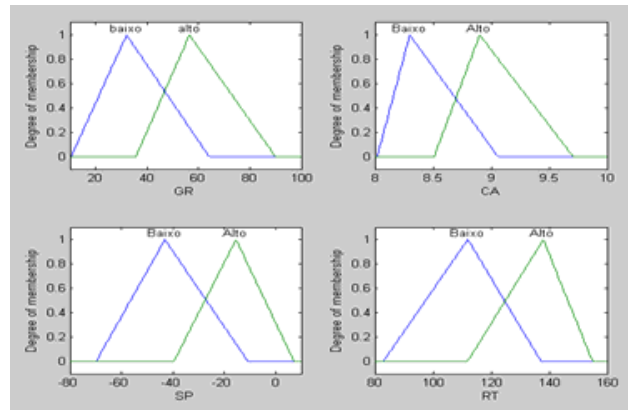

Figure 10. Funções de pertinência para após eliminação da função médio e a utilização do algoritmo ISODATA. 
Isto permitiu uma melhora significativa na estimativa tanto da porosidade como da permeabilidade, como pode ser verificado na Figura 8.

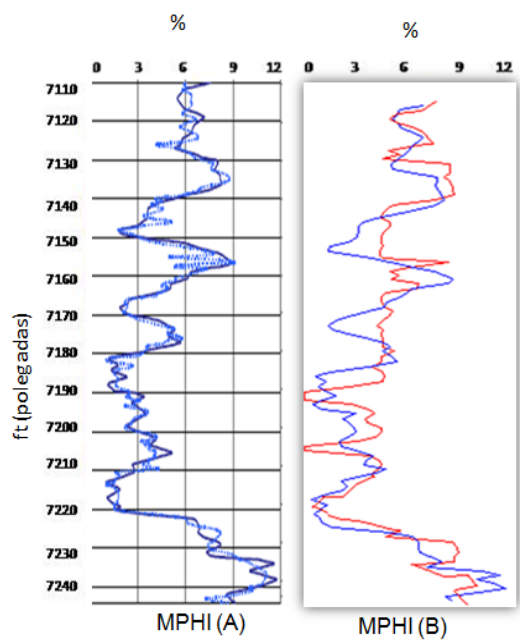

Figura 1. Comparação dos resultados da simulação de Richardson (1999), à esquerda, e os nossos, à direita, para a permeabilidade.

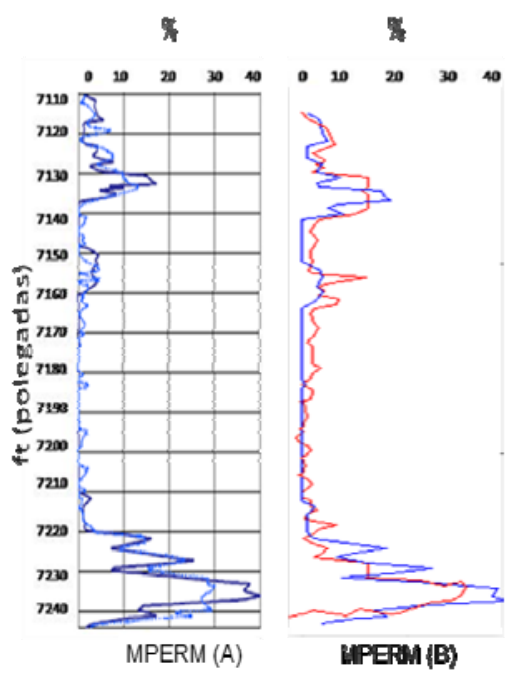

Figura 12. Comparação dos resultados da simulação de Richardson (1999), à esquerda, e os nossos, à direita, para a permeabilidade.

\section{Conclusões}

Através deste trabalho mostramos a viabilidade da técnica de simulação de perfis RMN utilizando a lógica fuzzy. A base da metodologia consistiu em verificar a influência do número de regras e de funções de pertinência nos resultados, com a faixa de abrangência sendo retirada da clusterização. Essa abordagem nos encaminhou a resultados não satisfatórios, como simulações dos perfis de permeabilidade e de porosidade em forma de caixas. Essa dificuldade foi superada com a divisão dos grupos usando o algoritmo ISODATA, o qual promove uma divisão de grupos (clusters) mais criteriosa. Sendo assim obtiveram-se dois grupos com melhores pares de classes, resultando em melhor ajuste nas saídas. Pode-se observar o alto potencial da lógica fuzzy em prever as saídas com uma escolha aleatória das faixas de abrangência das funções de pertinência de saída, desde que as entradas sejam criteriosamente agrupadas e classificadas. O baixo ajuste para a permeabilidade ocorreu devido à larga faixa de interseção entre as funções de pertinência escolhidas para a saída. Os resultados obtidos com a metodologia fuzzy foram coerentes com os resultados de Richardson (1999) e as análises visuais dos perfis de RMN. Isto comprova a eficácia da metodologia fuzzy atendendo ao objetivo de tornar-se uma ferramenta que possa ser utilizada para simulação de perfis de poços.

\section{Agradecimentos}

Agradecemos à FAPERJ pela bolsa de estudos oferecida e a Universidade Estadual do Norte Fluminense Darcy Ribeiro pelo suporte computacional.

\section{Referências}

Asquith, G. \& Gibson, C. 1982. Basic well log analysis for geologists. Oklahoma: AAPG, 216 p.

Chapman, S. 2006. Programação em MATLAB para Engenheiros. Tradução Técnica Flávio Soares Correa da Silva. Editora Thomson Learning, 2003 - São Paulo.

Coates, G.; Xiao, L. \& Prammer, M. 1999. Registros Eléctricos RMN, Principios y Aplicaciones. Halliburton Energy Services. 233p.

Mandani, E., H., 1974. Application of Fuzzy Algorithm for Control os Simple Dynamic plant. Proceedings of IEEE Control and Science.

MatLab. 2008. User's Manual.

Richardson, M. 1999. Using Conventional Wireline Logs to Generate Magnetic Resonance Imaging (MRI) Logs: A Feasibility Study. MSC. Thesis, West Virginia University, Morgantown, $99 \mathrm{pp}$.

Zadeh, L., Tanaka, K. \& Shimura, M. 1975. Fuzzy Sets and their Applications Congnitive and Decision Processes. Academic Press, New York, 496p. 